\title{
Bioequivalence of Topical Clotrimazole Formulations: An Improved Tape Stripping Method
}

\author{
Natalie Rae Parfitt ${ }^{1}$, Michael Skinner ${ }^{2}$, Charles Bon ${ }^{3}$, Isadore Kanfer ${ }^{1}$ \\ ${ }^{1}$ Division of Pharmaceutics, Faculty of Pharmacy, Rhodes University, Grahamstown, 6140, South Africa. \\ ${ }^{2}$ Biopharmaceutics Research Institute, Rhodes University, Grahamstown, 6140, South Africa. \\ ${ }^{3}$ Biostudy Solutions, Wilmington, USA.
}

Received, June 28, 2011; Revised, July 15, 2011; Accepted, August 30, 2011; Published, August 31, 2011.

\begin{abstract}
Purpose: Investigations were carried out to assess the use of tape stripping (TS) for the determination of bioequivalence of topical products containing 1\% clotrimazole. Methods: The study design involved the establishment of an appropriate application time, which was determined by conducting a dose duration study. Subsequently, two bioequivalence studies were conducted: i) using the brand (Canesten Topical - $1 \%$ clotrimazole cream) as both the test and the reference product and ii) comparing Canesten cream with a gel product containing the same concentration of clotrimazole (1\%). Each tape strip was individually analyzed for clotrimazole content using an HPLC method and Transepidermal Water Loss (TEWL) measurements were used to normalize the stratum corneum thicknesses between subjects. Results: The results of the TS investigations showed that, if the study is sufficiently powered, tape stripping may be used to determine bioequivalence according to the conventional bioequivalence limits of $0.8-1.25$, as well as detect formulation differences between different clotrimazole products. Conclusions: The data from this study provided compelling evidence that tape stripping has the necessary attributes and potential to be used as a tool for the bioequivalence assessment of topical clotrimazole and/or other topical formulations, thereby circumventing the need to undertake expensive and time-consuming clinical trials for such products.
\end{abstract}

This article is open to POST-PUBLICATION REVIEW. Registered readers (see "For Readers") may comment by clicking on ABSTRACT on the issue's contents page.

\section{INTRODUCTION}

The specialised nature of the stratum corneum makes it an efficient barrier to foreign substances, including drug molecules. Therefore, cutaneous drug absorption is a slow and complex process in which stratum corneum penetration is the rate limiting step. The rate and extent of stratum corneum penetration by a drug compound depends on the formulation and presence of penetration enhancing/retarding excipients. Therefore the clinical outcomes of a product rely greatly on the components and quality of the formulation. Hence, establishing bioequivalence between topical products is crucial in order to ensure that patients receiving multisource drug products are assured of the same efficacy and safety as the brand product.

Since locally acting topical formulations do not target the systemic circulation, conventional methods of assessing bioequivalence by measuring concentrations of drug in blood samples are not appropriate. With the exception of topical corticosteroid preparations (1), the current regulatory guidelines require comparative clinical trials to be carried out to show bioequivalence between topical products. As these studies are very expensive and time consuming, the development of a more direct and relatively rapid and inexpensive method for determining bioequivalence of topical products is required.

The tape stripping (TS) technique involves the sequential removal of microscopic layers $(0.5-1 \mu \mathrm{m})$ of the stratum corneum by placing a strip of adhesive tape onto the skin surface with uniform pressure, and then removing the tape strip (2). By harvesting stratum corneum samples from skin previously exposed to a topical product, drug uptake into the stratum corneum can be measured in vivo (3-9).

Corresponding Author: I Kanfer, Faculty of Pharmacy, Rhodes University, Grahamstown, 6140, South Africa; E-Mail: I.Kanfer@ru.ac.za 
The TS technique is recognized as relatively simple and inexpensive, and has been described as minimally invasive (10) and virtually painless (2). Several reports have described the use of TS to assess bioavailability of topical products, and it has been investigated as a method for the determination of bioequivalence of some topical products (2-9).

In 1998 the FDA published an industry guidance (11) which described the TS method for the assessment of bioequivalence of topical products. The study design endeavored to simulate the absorption and elimination phases seen in an oral pharmacokinetic study so that parameters analogous to those used in conventional $\mathrm{BE}$ studies could be determined e.g. AUC and $\mathrm{C}_{\max }$. This was achieved by using an 8 point profile (time vs. drug amount) which represented the "uptake" and "elimination" phases of drug absorption. The use of this protocol produced some promising results (12), however, in 2002 the FDA withdrew the guidance since major concerns were raised regarding the adequacy of the TS method to assess topical products that did not target the stratum corneum and the lack of reproducibility of the method between laboratories (13).

Since the withdrawal of the guidance in 2002, attempts have been made to improve the TS method and minimize the variability associated with it. The idea of using the AUC or $\mathrm{C}_{\max }$ of an 8 point profile has largely been rejected (14) and instead a comparison of the total amount of drug found in the stratum corneum has been suggested as an alternative to those parameters. By comparing the total amount of drug present in the stratum corneum at a single time point, differences have been shown between cream and ointment formulations (8). However, this is a relatively rugged approach and assumes that the amount of stratum corneum harvested from each subject is the same. It also depends greatly on the chosen dose duration, i.e. application time or duration of the application. An adaptation of this approach is the use of 2 time points, 1 representing the "uptake" phase and 1 representing the "clearance" phase (7, 15). This approach also uses the total amount of drug found in the stratum corneum as an assessment parameter. However, by including Transepidermal Water Loss (TEWL) measurements, stratum corneum thicknesses from individual human subjects can be determined (16), whereafter individual differences in skin thickness between subjects can be normalized. Another approach, described by Herkenne et al (4), involves the analysis of the drug content of each tape strip in conjunction with the standardization of stratum corneum thickness between subjects using TEWL measurements. This provides detailed information regarding the amount of drug present in the stratum corneum as well as the extent of drug penetration.

Clotrimazole (CLZ) is an anti-fungal agent which is used topically to treat cutaneous infections such as candidiasis, dermatophytoses, tinea vesicolor and erythrasma. CLZ has its site of action in the stratum corneum where the pathogens causing these conditions reside. Since the TS technique involves sampling the stratum corneum it was considered appropriate to investigate this method as a possible tool for the determination of bioequivalence of topical CLZ products.

In this work, a single dose left on the skin for a specific period of time (dose duration) was used, and each tape strip was individually assayed and the stratum corneum depth was normalized using TEWL measurements (4). In addition, a novel approach of conducting a preliminary dose duration study to determine an appropriate dose duration (time drug left on skin) for the bioequivalence investigations was used. This approach was based on the FDA Human Skin Blanching Assay (HSBA) guidance (1) (the pharmacodynamic method of determining bioequivalence between topical corticosteroids). In the HSBA it is necessary to ensure that the pivotal study is carried out at the most sensitive part of the dose-response curve. Therefore, in accordance with the FDA guidance on topical corticosteroids, a pilot study which uses the $\mathrm{E}_{\max }$ model to determine the dose duration where the maximum sensitivity can be expected, i.e. the $\mathrm{ED}_{50}$, was determined for clotrimazole.

\section{METHODS}

\section{Chemicals}

CLZ (99\%) was purchased from Sigma-Aldrich (Atlasville, South Africa) and HPLC-grade acetonitrile was purchased from Romil Ltd (Waterbeach, Cambridge, UK). The water was purified using a Milli-Q system (Millipore, Bedford, MA, USA).

\section{Formulations}

The studies involved the use of two commercially available products each containing $1 \% \mathrm{~m} / \mathrm{m}$ CLZ. Canesten is the innovator brand of CLZ and 
therefore Canesten Topical cream (Bayer, Germany) was used as the reference product. Candid gel (Glenmark, India) was used as a second test product

\section{Study design}

A dose duration study, which is a novel approach for a non-corticosteroid drug, was employed to determine a suitable dose duration for the bioequivalence study. The bioequivalence studies were subsequently conducted using a dose duration equal to the ED50 determined from the Emax model. For the bioequivalence investigations, Canesten Topical cream was used as both test and reference products to determine if the method was capable of showing bioequivalence. Subsequently, Canesten Topical cream was also compared to a $1 \%$ gel formulation ( $2^{\text {nd }}$ test product) to determine if the method could detect formulation differences if indeed differences in bioavailability did exist.

\section{Dose duration study}

Ten volunteers ( 1 male, 9 females) between the ages of 19 and 28 (mean 22 years) were enrolled in the dose duration study. Within this group, 7 subjects were Caucasian, 2 were of Indian or Malay descent and 1 black subject was included. Written informed consent was obtained from each volunteer before each study. The research with human subjects followed the recommended guidelines as set out in the Declaration of Helsinki (1964) and associated amendments (17). The study protocol was approved by the Rhodes University Ethical Standards Committee (Grahamstown, South Africa).

Eight sites $(2 \times 2 \mathrm{~cm})$ were demarcated on the left arm of each subject using a template prepared by reinforcing the non-adhesive side of Opsite Flexifix (Smith \& Nephew Medical Ltd, London, England) with Scotch Magic ${ }^{\mathrm{TM}}$ Tape (no.810, 3M, USA). Seven of the sites were used for product application and 1 site was reserved as a blank site. A volume equivalent to an amount of $15.2 \mathrm{mg}$ Canesten Topical cream ( $0.152 \mathrm{mg}$ of CLZ) using a calibrated Eppendorf pipette was dispensed to each site at time zero. A pre-weighed glass rod was used to spread the product within the area delineated by the template and the rod was weighed following spreading.

Each application site was exposed to the formulation for different dose durations $(0.25 \mathrm{hrs}$, $0.5 \mathrm{hrs}, 1 \mathrm{hr}, 2 \mathrm{hrs}, 4 \mathrm{hrs}, 6 \mathrm{hrs}$ or $8 \mathrm{hrs}$ ). Once the necessary dose duration had elapsed, 2 cotton swabs were used to remove the residual formulation from the site and the TS procedure commenced. The tape stripping procedure involved the use of 15 pre-weighed tape strips per site. The tape strips were prepared in-house using Sellotape Original (Henkel Consumer Adhesives, Johannesburg, South Africa). The direction of stripping was rotated $(\mathrm{N}, \mathrm{W}, \mathrm{S}, \mathrm{E})$ to ensure uniform removal of stratum corneum (18). After stripping, each tape was immediately weighed using a Precisa 180A balance (Precisa Balances Ltd., Geneva, Switzerland).

The blank site underwent the same TS procedure, but in addition, TEWL measurements were taken after each stripping using a Delfin VapoMeter (Delfin Technologies Ltd., Finland) in order that the thickness of each subject's stratum corneum could be calculated. After TS, the tape strips used on the blank site were used for spiking with standard solutions of CLZ to prepare a calibration curve for subsequent sample analysis by HPLC.

The tape strips from both the blank and drug application sites were placed individually into $2 \mathrm{ml}$ microcentrifuge tubes. Methanol $(500 \mu \mathrm{l})$ was added to the tubes and the samples were vortexed for 1 minute using an Eppendorf MixMate (model PCB-08, Eppendorf, Hamburg, Germany) at 2300 rpm and then centrifuged for 10 minutes (Eppendorf Centrifuge 5415, Hamburg, Germany) at $1300 \mathrm{rpm}$. The resulting extract was injected (10 $\mu \mathrm{l})$ into an HPLC system consisting of a Luna $5 \mu \mathrm{m}$ (C18) $100 \AA 150 \times 4.60 \mathrm{~mm}$ (Phenomenex) column (maintained at $40^{\circ} \mathrm{C}$ ) perfused at a flow rate of 1 $\mathrm{ml} / \mathrm{min}$ by a mobile phase consisting of acetonitrile and $0.005 \mathrm{M}$ ammonium acetate $(\mathrm{pH}=7.6)$ in the ratio 65:35. The CLZ peaks were monitored at a wavelength of $210 \mathrm{~nm}$.

The stratum corneum thickness $(\mathrm{H})$ for each individual was determined from the following equation (16):

$$
\text { 1/TEWL } \mathrm{T}_{\mathrm{x}}=\mathrm{H}-\mathrm{x} / \text { K.D. } \Delta \mathrm{C} \quad(\text { Equation 1) }
$$

where $\mathrm{TEWL}_{\mathrm{x}}$ is the transepidermal water flux after a total of $\mathrm{x} \mu \mathrm{m}$ of stratum corneum has been removed after consecutive stripping of the blank (unmedicated) site; $\mathrm{H}$ is the total stratum corneum thickness, $\mathrm{x}$ is the cumulative stratum corneum thickness removed after consecutive tape strips i.e. partial of $\mathrm{H}$; $\mathrm{K}$ is the partition coefficient of water 
from the stratum corneum to viable tissue; D is the average apparent diffusivity of water in the stratum corneum, and $\Delta \mathrm{C}$ is the difference of water concentration across the membrane.

The total SC thickness $(\mathrm{H})$ calculated from the blank site for a subject is used as that individual's $\mathrm{SC}$ thickness. The SC thickness on each strip from the blank site (i.e. the layer of SC adhering to each tape strip) is calculated from the mass of skin removed, the area stripped (i.e. area of the strip of tape used to do the stripping) and an estimated SC density of $1000 \mathrm{mg} / \mathrm{mL}$ where the mass is the product of the area of the tape strip, the thickness of the SC layer removed by the tape strip and the density. According to equation 1, 1/TEWL data (y-axis) obtained from the blank site after each tape strip is then plotted against the corresponding cumulative SC thickness ( $\mathrm{x}$ ) values obtained as described above. The data were then fitted using linear regression analysis to obtain the equation to describe the plot and the $\mathrm{x}$-intercept, which is the theoretical total SC thickness $(\mathrm{H})$ for that particular subject, was obtained (Figure 1).

When processing the data from the application sites, the first tape strip was not included in the analysis because it is highly likely that it would contain some unabsorbed drug product remaining on the application site on the skin that had not been completely removed by the cotton wool swabs.
The amount of drug per strip and the fraction of stratum corneum removed were calculated for each of the remaining 14 individual strips per site. Thereafter, the AUC from a plot of the amount of drug per strip vs $\mathrm{x} / \mathrm{H}$ (cumulative fraction of stratum corneum removed) was calculated for each medicated site using the trapezoidal rule (Figure 2). These AUC values can then be used to determine dose-response profiles or to compare the bioavailability between test and reference products to assess bioequivalence using appropriate statistical procedures and regulatory acceptance criteria.

The dose - response profile was constructed using mean AUC values obtained for each dose duration ( $\mathrm{n}=10)$. An AUC value of 0 was assumed at time $=0$ hours as no CLZ penetration could have occurred.

This 8-point profile was fitted to the Emax model and the ED50 was calculated using GraphPad Prism software Version 4 (GraphPad Software, San Diego, California, (USA).

\section{Bioequivalence study}

The bioequivalence study was conducted using 13 subjects ( 9 females and 4 males) between the ages of 19 and 30 years (mean 25 years). Within this group there were 4 Caucasian and 4 black subjects, 4 of Indian or Malay descent and 1 Asian subject.

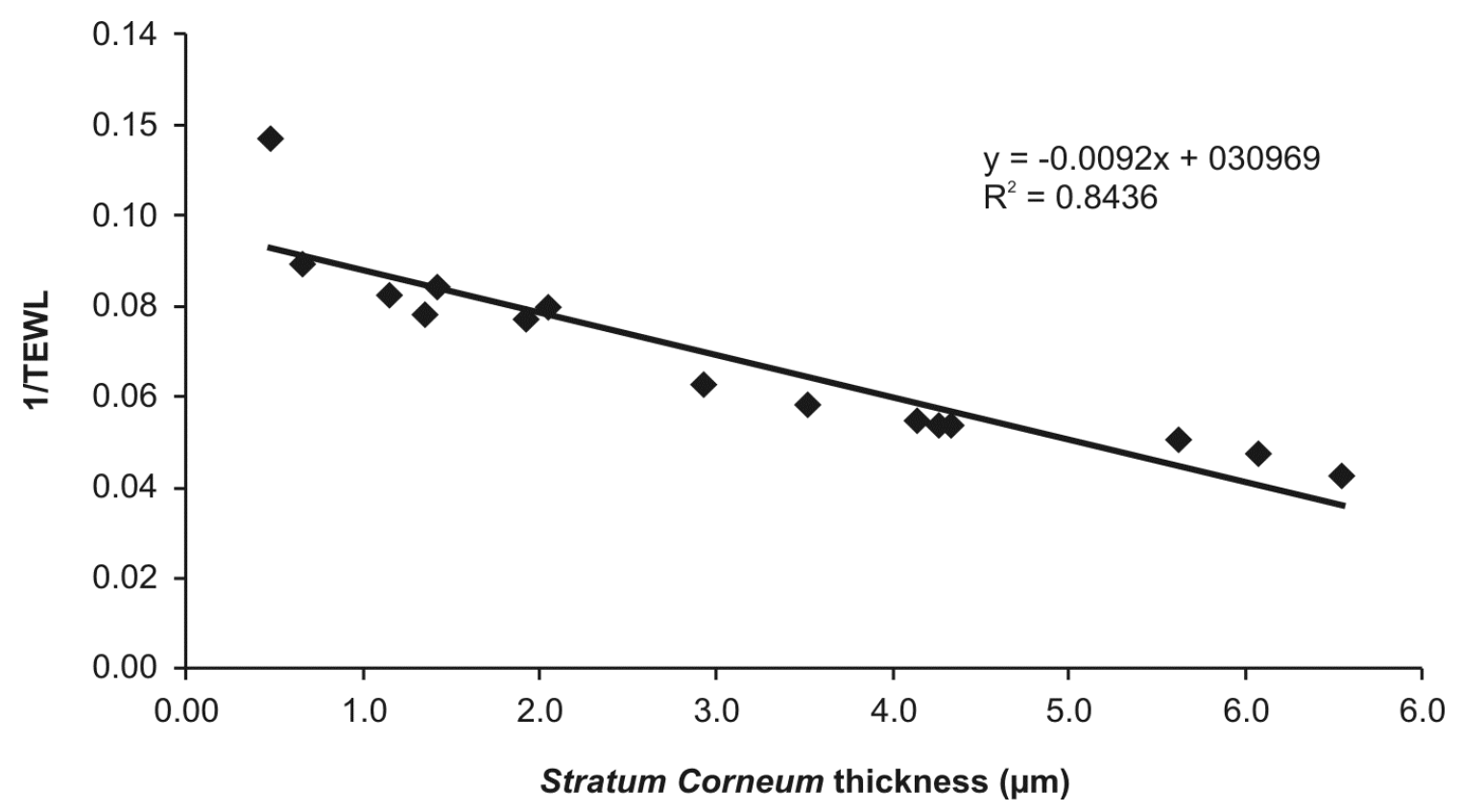

Figure 1. Plot of 1/TEWL vs cumulative SC thickness (x) used to determine the theoretical skin thickness $(H)$ from the $\mathrm{x}$-intercept of the linear regression line. 


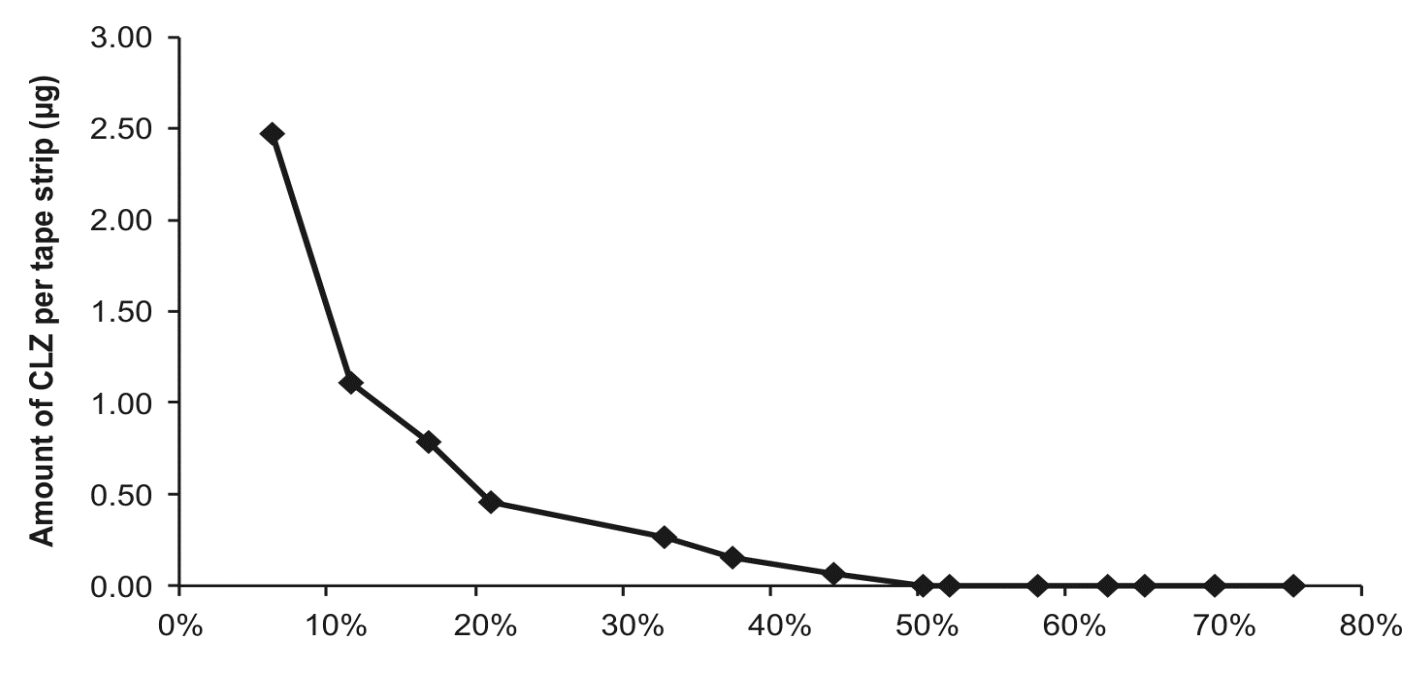

Cumulative fraction of Stratum Corneum $(\mathrm{x} / \mathrm{H})$

Figure 2. Plot of amount of CLZ per tape strip $(\mu \mathrm{g})$ vs cumulative fraction of SC removed (x/H\%) to provide a drug penetration profile for the determination of AUC values.

Canesten Topical cream was used as both the test and the reference products in order to determine whether the method was capable of confirmimg bioequivalence using the same product. Four application sites and 1 blank site were delineated on the left arm of each subject using a method involving the use of 2 different templates per site. The designation of the 2 "test" and 2 "reference" sites was randomized between individuals.

As illustrated in the figure below, the first template was used to demarcate an area of $2.2 \times 2.2$ $\mathrm{cm}$ on the skin (Figure 3 (i)) and then a permanent marker was used to mark the corners, thereby outlining an area of $2 \times 2 \mathrm{~cm}$ (Figure 3 (ii)). Canesten Topical cream $(15.2 \mathrm{mg})$ was then applied within the bounds of the marked areas and left in contact with the skin for 15 minutes (as determined by the dose duration study) before removal (Figure 3 (iii)) with 2 cotton swabs. Although the calculated ED50 was $10.8 \mathrm{~min}$, since it takes approximately 10 min to carry out the TS procedure, a dose duration of $15 \mathrm{~min}$ was selected. Before TS commenced, a fresh $2 \times 2 \mathrm{~cm}$ template was applied to accurately delineate the sampling area (Figure 3).

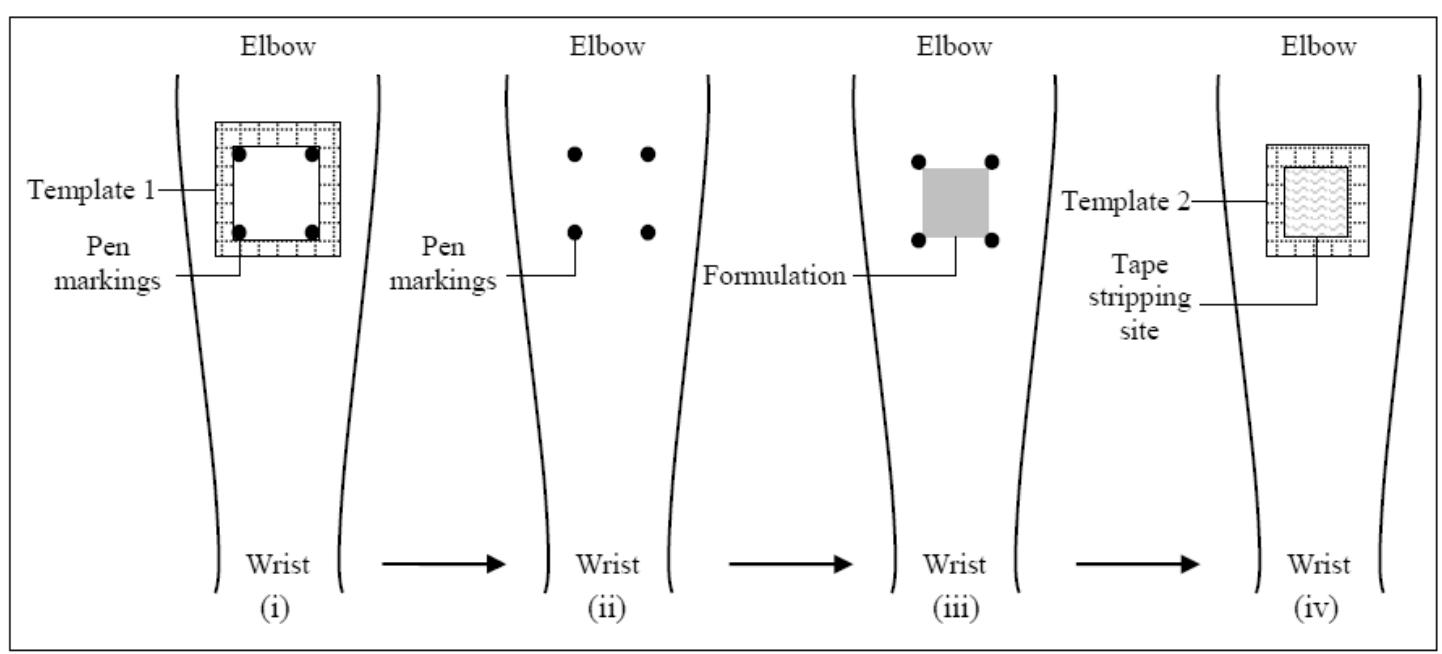

Figure 3. The double template design used to prevent template contamination 
This design ensured that the template used for the TS procedure was not contaminated with any formulation that may have spread laterally and consequently possibly have been absorbed by the template during the dosing period.

The TS procedure and the TEWL measurements taken at the blank site were carried out as previously described. The normalization of stratum corneum depth and calculation of AUC were conducted as for the dose duration study. An $\mathrm{AUC}_{\text {test }}$ and $\mathrm{AUC}_{\text {reference }}$ value was determined for each subject by taking the mean $(n=2)$ of the AUC values for each "product". As each subject received both the test and the reference product the study was considered to have a paired / crossover design. No guidelines for the analysis of TS data are available, therefore both $\ln$-transformed and untransformed data were used for the assessment of bioequivalence.

EquivTest 2.0 (Statistical Solutions Ltd, Ireland) software was used to calculate the $90 \%$ confidence interval $\left(\mathrm{CI}_{90 \%}\right)$ for the $\mathrm{AUC}_{\text {test }} / \mathrm{AUC}_{\text {reference }}$ ratios for both sets of data. For the untransformed data, the point estimate was calculated by dividing the mean $\mathrm{AUC}_{\text {test }}(\mathrm{n}=10)$ value by the mean $\mathrm{AUC}_{\text {reference }}$ $(\mathrm{n}=10)$ value and the $\mathrm{CI}_{90 \%}$ was determined using Fieller's/Locke's method described in the FDA Guidance (1). The CV\% associated with the ratio was calculated using the following equation for untransformed data:

$$
\mathrm{CV} \%=\sqrt{ }(\mathrm{MSE} / \text { mean }) * 100 \quad \text { (Equation 2) }
$$

In order to determine the number of subjects required for $80 \%$ statistical power the method described for "raw data from a cross over study design" by Chow and Wang (19) was used. For the In-transformed data, the Schuirmann two one-sided test (TOST) (20)was used to calculate the $\mathrm{CI}_{90 \%}$ and the point estimate. The following equation was used to determine the $\mathrm{CV} \%$ associated with the ratio using $\ln$ - transformed data:

$$
\left.\mathrm{CV} \%=\sqrt{ }\left(\mathrm{e}^{\mathrm{MSE}}-1\right) * 100 \quad \text { (Equation } 3\right)
$$

The statistical power of the ln-transformed data was calculated using the MS Excel 2003 spreadsheet accompanying the 5th edition of Pharmaceutical Statistics: Practical and Clinical Applications (21).

\section{Bioinequivalence confirmation study}

As this was a "proof of concept" study only 7 subjects (6 females and 1 male) were used to provide preliminary data. The average age of the subjects was 28 years and 3 black, 1 Asian, 2 subjects of Indian or Malay descent and 1 Caucasian were included in this study.

This study design was based on previous studies performed in our laboratory (18) where only three sampling sites and one blank site were involved. The templates described in the previous bioequivalence study were applied to the ventral surface of the left forearm to demarcate the application sites. Canesten Topical cream was applied to two of the three sampling sites. One of the doses of Canesten Topical cream applied to these sites was designated the reference product $(\mathrm{R})$ and the other as test product one $\left(\mathrm{T}_{1}\right)$. Candid 1\% CLZ gel was applied to the remaining site and was designated test product two $\left(\mathrm{T}_{2}\right)$. The application of the products was randomized. As previously stated and determined from the dose duration study, each site was exposed to the formulation for 15 minutes, after which the residual formulation was removed and the site was tape stripped. As before, individual stratum corneum depth was assessed using a blank site and TEWL measurements.

\section{RESULTS}

\section{Dose duration study}

The results of the dose duration study showed rapid CLZ penetration within the first 2 hours, after which the rate of penetration appeared to level out, indicating that steady state had been achieved (Figure 4). The data were fitted to the Emax model with $\mathrm{R}^{2}=0.9648, \mathrm{E}_{\max }=89.06$ and $\mathrm{ED} 50=0.18 \mathrm{hr}$ (10.8 mins). Based on the ED50, a dose duration of 15 minutes was chosen for the bioequivalence studies.

\section{Bioequivalence study}

The results of this study (Table 1) suggest that regardless of whether ln-transformed or untransformed data are used for analysis, if sufficiently powered, TS is capable of showing bioequivalence between CLZ products that are truly bioequivalent i.e. when the innovator product is used as both the test and reference product. 


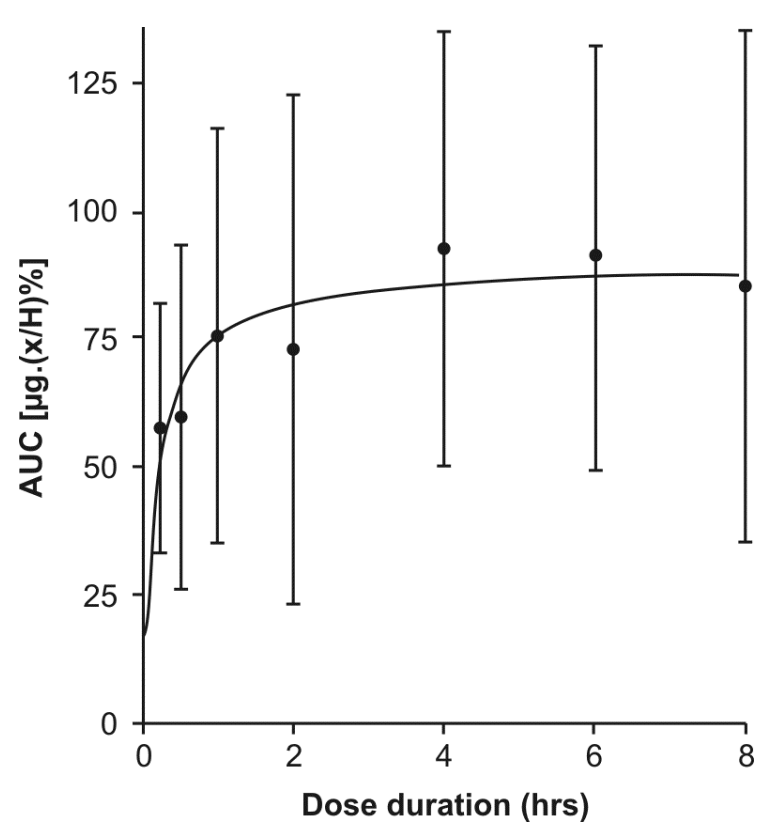

Figure 4. Dose duration profile for Canesten Topical cream $(n=10)$.

\begin{tabular}{lcc}
\hline \multicolumn{3}{c}{ Table 1. Summary of results for the bioequivalence study } \\
\hline & $\begin{array}{c}\text { Untransformed } \\
\text { Data }\end{array}$ & $\begin{array}{c}\text { Transformed } \\
\text { Data }\end{array}$ \\
\hline $\mathrm{n}$ & 13 & 13 \\
$\mathrm{AUC}_{\text {test }} / \mathrm{AUC}_{\text {reference }}$ & 0.94 & 0.97 \\
$\mathrm{CI}_{90 \%}$ & $0.82-1.08$ & $0.82-1.13$ \\
Bioequivalence? & & \\
$(0.8-1.25)$ & Yes & Yes \\
$\mathrm{CV} \%$ & $23.62 \%$ & $23.40 \%$ \\
$\mathrm{G}$ & 0.0399 & $\mathrm{n} / \mathrm{a}^{* *}$ \\
Power & $\mathrm{n} / \mathrm{d}^{*}$ & $47.24 \%$ \\
$\mathrm{n}$ required for $80 \%$ power & 19 & 21 \\
\hline $\mathrm{n} / \mathrm{d}^{*}:$ not determined, $\mathrm{n} / \mathrm{a}^{* *}:$ not applicable. \\
\hline \multicolumn{2}{l}{} \\
\hline
\end{tabular}

According to the untransformed data, only 6 additional subjects $(n=19)$ would be required to achieve a statistical power of $80 \%$ whereas using $\ln$-transformed data, 8 additional subjects $(n=21)$ would be required.

\section{Influence of expanding the bioequivalence limits}

In view of the fact that the practical application of topical dosage forms such as CLZ is relatively imprecise in the clinical setting, the stringency of the current acceptance criteria may be unnecessary. Hence, the effect of widening the BE limits and its implications with respect to subject numbers and the associated study power was investigated using the current data. It can be seen (Table 2) how increasing the limits for the $\mathrm{CI}_{90 \%}$ of the ratio substantially reduces the sample size required. When the conventional limits are used, 19 or 21 subjects are required for an $80 \%$ power. On the other hand, as few as 9 subjects (using transformed data) may provide a satisfactory outcome.

\begin{tabular}{llc}
\hline \multicolumn{3}{l}{ Table 2. Effect of widening the bioequivalence limits } \\
\hline $\begin{array}{l}\text { Bioequivalence } \\
\text { limits }\end{array}$ & Sample size required for $80 \%$ power \\
\hline & $\begin{array}{c}\text { Untransformed } \\
\text { data }\end{array}$ & $\begin{array}{c}\text { Transformed } \\
\text { data }\end{array}$ \\
\hline $0.8-1.25$ & 19 & 21 \\
$0.75-1.33$ & 14 & 13 \\
$0.7-1.43$ & $<13$ & 9 \\
\hline
\end{tabular}

As can be seen above (Table 3), in the Canesten vs. Canesten investigation the $T_{1} / R$ ratio was found to be close to 1 for both the transformed and the untransformed data. However, because a sample size of only 7 subjects was used, the variability associated with the study was relatively high and the $\mathrm{CI}_{90 \%}$ fell outside of the BE limits for both products. The small sample size also contributed to the study having a very low statistical power. In order to increase the statistical power of the study to $80 \%$, approximately $30-45$ subjects would be required. By increasing the number of subjects it is possible that the $\mathrm{CI}_{90 \%}$ would narrow around the $\mathrm{T}_{1} / \mathrm{R}$ point estimate and confirmation of $\mathrm{BE}$ between the bioequivalent applications might be shown.

In contrast, the ratio found by comparing the gel with the reference product, Canesten (i.e. $T_{2} / R$ ) was approximately 2 for both sets of data. This indicated that the rate and extent of CLZ penetration from the gel was double that of the cream formulation. This clearly showed that these products were not bioequivalent. Because the point estimate was so much greater than 1, the probability of this study showing BE was completely unlikely regardless of the sample size used.

\section{Influence of expanding the bioequivalence limits}

As in the bioequivalence study, the effect of widening the bioequivalence limits was investigated for the $\mathrm{T}_{1} / \mathrm{R}$ study. Although the $\mathrm{CI}_{90 \%}$ for $T_{1} / R$ did not fall within the BE limits of $0.75-$ 
1.33 or even between $0.7-1.43$, the number of subjects required to confirm these results was calculated.

Table 4 above shows how the number of subjects required to confirm the study results decreases as the BE limits are widened. As the point estimate and $\mathrm{CI}_{90 \%}$ for the $\mathrm{T}_{2} / \mathrm{R}$ study was so far out of the BE limits, calculating the sample size required for various bioequivalence limits would have been meaningless.

In addition, skin penetration profiles for each product were obtained. However, since all points have different $\mathrm{x} / \mathrm{H}$ values, true mean profiles cannot be determined. These mean skin penetration profiles (Figure 5) are therefore simply an approximate comparative representation of the various drug product profiles within the skin and are useful for visualization purposes only. Data points for these profiles were determined by calculating the mean of both the $x$ values $(x / H)$ and $y$ values (amount per strip) for all subjects for data points with $\mathrm{x}$-values within specified sequential ranges over the full $\mathrm{x} / \mathrm{H}$ range of 0 to 1 .

\begin{tabular}{lllll}
\hline \multicolumn{5}{l}{ Table 3: Summary of results for the bio-inequivalence study } \\
\hline
\end{tabular}

\begin{tabular}{lll}
\hline \multicolumn{3}{l}{ Table 4 Effect of widening the bioequivalence limits on the power of the $\mathbf{T}_{\mathbf{1}} / \mathbf{R}$ study } \\
\hline Bioequivalence limits & Sample size required for $80 \%$ power & Transformed data \\
\hline $0.8-1.25$ & Untransformed data & 44 \\
$0.75-1.33$ & 29 & 23 \\
$0.7-1.43$ & 22 & 14 \\
\hline
\end{tabular}

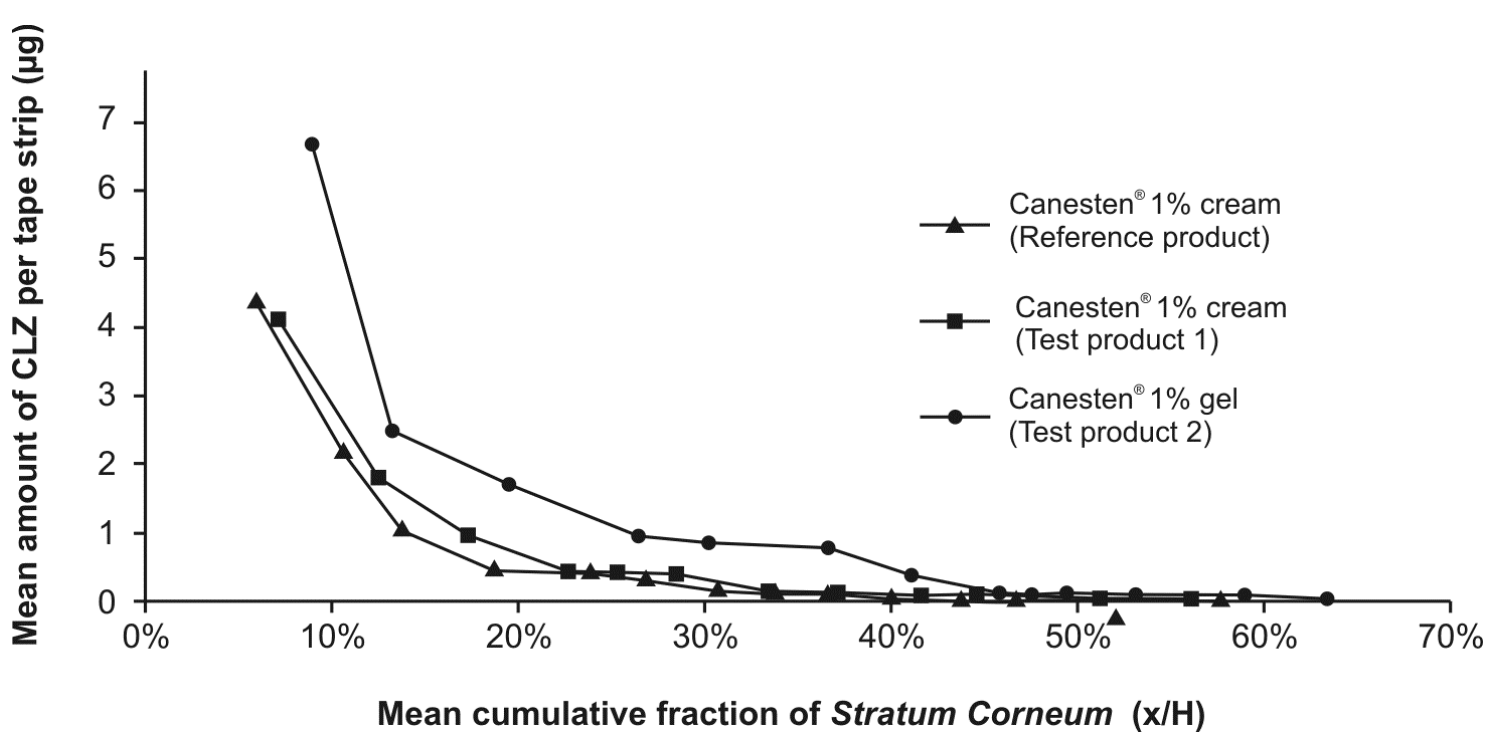

Figure 5. Comparative skin penetration profiles for various clotrimazole products $(n=7)$ 


\section{DISCUSSION}

A major consideration when using a single dose duration for a TS study is the choice of actual time the dose is left on the skin. In other studies $(4,7-9,14,22)$, the choice of dose duration has generally been unsubstantiated. Sampling when the concentration of drug in the stratum corneum is at steady state is likely to mask differences in formulations, thus it is important to have a validated method of ensuring that the chosen dose duration falls on a sensitive part of a dose response relationship, such as a plot of the dose duration $v s$. drug penetration profile.

In order to determine a dose duration which will provide the necessary discriminatory power to identify significant differences or equality between products, the approach employed in the FDA Human Skin Blanching Assay (HSBA) guidance (1) (the pharmacodynamic method of determining bioequivalence between topical corticosteroids) was used. From the dose duration study and fitting the profile to the $\mathrm{E}_{\max }$ model, a dose duration of 15 minutes was determined to be appropriate for the intended bioequivalence studies.

As there are presently no regulatory guidelines regarding the analysis of TS data, the bioequivalence limits used in this study (i.e. 0.8 -1.25) were based on those described in the guidance used for bioequivalence studies involving oral products (23). For oral products, the clinical relevance of these limits has been demonstrated over many years and hence the use of these limits has become internationally accepted. In the majority of cases, empirical clinical evidence has shown that oral products deemed to be bioequivalent according to these limits have the same therapeutic outcomes as their innovator counterparts (24).

However, the clinical relevance of applying these limits to BE studies involving topical products is questionable. As the use of oral (finite dose) and topical dosage forms (variable dose), as well as the pharmacokinetics associated with each are virtually incomparable, it is highly unlikely that the limits of $0.8-1.25$ have any clinical significance for locally acting topical products. This was recognized in the draft DPK guidance issued by the FDA, where the limits for the BE parameter $\mathrm{A}_{\max }$ were relaxed to $0.7-1.43$ (11).

The results of the bioequivalence study showed that TS can be used to determine bioequivalence, according to the conventional limits, if the study is sufficiently powered. In this study, the results attained using the transformed and untransformed data were virtually the same, showing that both methods of analysis may be considered acceptable for the assessment of TS data.

Although, the small sample size used in the bioinequivalence study resulted in the power of the studies being very low and the BE assessments being inconclusive, this small investigative study was able to demonstrate that the TS method is capable of detecting both differences and similarities between CLZ formulations, albeit not being able to confirm bioequivalence in this instance. Considering the results from the previous Canesten vs. Canesten study, it is possible that an increase in the number of subjects would provide more informative and realistic results. However, the data suggest that, regardless of the increase in subject numbers, the outcome of the Candid gel vs. Canesten Topical cream study would remain unchanged.

Whilst the data obtained from this study may be inconclusive because of the low number of subjects, the vast differences found between the cream and gel formulations suggest the potential of the method to detect formulation differences if such differences truly exist.

A comparison of the results obtained using untransformed and $\ln$-transformed data shows that both approaches are satisfactory for the analysis of TS data. However, the relevance of using ln-transformed data for dermal BE studies is as yet undetermined. It is possible that contrary to $\mathrm{BE}$ data of oral products, TS data are normally distributed and do not require logarithmic transformation.

Finally, in view of the differences in skin thickness between subjects, it is important to normalize those data using TEWL measurements (16).

\section{CONCLUSIONS}

These studies have shown that, if the method is carried out strictly in accordance with the specified protocol and properly powered, it is possible to use the TS method to determine bioequivalence between topical CLZ products. The determination of an appropriate dose duration as well as standardization of skin thicknesses between subjects are important considerations. The results are heartening because they suggest that TS has the 
potential to be used in a regulatory setting as a tool for the assessment of bioequivalence between topical CLZ products. As TS is a more direct method of assessing BE than comparative clinical trials, as well as being less costly and time consuming, it would be extremely advantageous to be able to use this method instead.

Because the site of action for antifungals such as CLZ is the stratum corneum and the TS method takes samples directly from this tissue, this technique is particularly appropriate for the assessment of these formulations. Therefore, by using CLZ as a model compound, these studies have shown that the TS method has the potential to be used as an assessment tool not only for CLZ formulations, but also for other antifungal preparations.

By assaying each tape strip individually and normalizing the amount of stratum corneum removed, through the use of TEWL measurements, detailed information regarding the amount and extent of CLZ penetration was obtained. This approach was different from some other TS studies where the total amount of drug present in the stratum corneum was used as the parameter for bioequivalence assessment and the distribution of the drug throughout the stratum corneum layer was not considered. However, it is recommended that data describing not only the total amount but also the distribution of the drug through the stratum corneum should be used when assessing topical formulations. From these TS studies, it was clear that the CLZ was not uniformly distributed throughout the stratum corneum and it is possible that the distribution of drug throughout the stratum corneum will affect the efficacy of the product. Therefore, by considering the extent of drug penetration (AUC of the amount CLZ/tape strip vs. relative stratum corneum depth profile) as an indicator of bioavailability using a pre-determined dose duration and correcting for differences in thickness of stratum corneum between subjects, this parameter is clearly appropriate to determine $\mathrm{BE}$ of such products.

\section{ACKNOWLEDGEMENTS}

The authors (IK) gratefully acknowledge financial support and a post-graduate scholarship (NRP) from the National Research Foundation, South Africa.

\section{REFERENCES}

1. US Department of Health and Human Services, Food and Drug Administration Center for Drug Evaluation and Research. Guidance for Industry: Topical Dermatological Corticosteroids: In vivo Bioequivalence. Rockville: Food and Drug Administration; 1995.

2. Herkenne C, Alberti I, Naik A, Kalia YN, Mathy F, Preat V, et al. In vivo methods for the assessment of topical drug bioavailability. Pharm Res, 25(1):87-103, 2008.

3. Herkenne C, Naik A, Kalia YN, Hadgraft J, Guy RH. Dermatopharmacokinetic prediction of topical drug bioavailability in vivo. J Invest Dermatol, 127(4):887-894, 2007

4. Herkenne C, Naik A, Kalia YN, Hadgraft J, Guy RH. Ibuprofen transport into and through skin from topical formulations: In vitro-in vivo comparison. J Invest Dermatol, 127(1):135-142, 2007.

5. Pershing LK, Corlett JL, Nelson JL. Comparison of dermatopharmacokinetic vs. clinical efficacy methods for bioequivalence assessment of miconazole nitrate vaginal cream, $2 \%$ in humans. Pharm Res, 19(3):270-277, 2002.

6. Pershing LK, Corlett J, Jorgensen C. In vivo pharmacokinetics and pharmacodynamics of topical ketoconazole and miconazole in human stratum corneum. Antimicrob Agents Chemother, 38(1):90-95, 1994.

7. Navidi W, Hutchinson A, N'Dri-Stempfer B, Bunge AL. Determining bioequivalence of topical dermatological drug products by tape-stripping. J Pharmacokinet Pharmacodyn, 35(3):337-348, 2008.

8. Benfeldt E, Hansen SH, Vølund A, Menne T, Shah VP. Bioequivalence of topical formulations in humans: Evaluation by dermal microdialysis sampling and the dermatopharmacokinetic method. J Invest Dermatol, 127(1):170-178, 2007.

9. Lodén $\mathrm{M}$, Akerström U, Lindahl K, Berne B. Bioequivalence determination of topical ketoprofen using a dermatopharmacokinetic approach and excised skin penetration. Int J Pharm, 284(1-2):23-30, 2004.

10. Lademann J, Jacobi U, Surber C, Weigmann H.-J., Fluhr J. The tape stripping procedure - evaluation of some critical parameters. Eur J Pharm Biopharm. 72(2):317-323, 2009.

11. U.S. Department of Health and Human Services, Food and Drug Administration, Center for Drug Evaluation and Research. Guidance for Industry Topical Dermatological Drug Product NDAs and ANDAs - In vivo Bioavailability, Bioequivalence, In vitro Release, and Associated Studies. Rockville: Food and Drug Administration; 1998.

12. Pershing LK, Nelson JL, Corlett JL, Shrivastava SP, 
Hare DB, Shah VP. Assessment of dermatopharmacokinetic approach in the bioequivalence determination of topical tretinoin gel products. J Am Acad Dermatol, 48(5):740-751, 2002.

13. Food and Drug Administration, HHS. Dermatological Drug Product NDAs and ANDAs - In Vivo Bioavailability, Bioequivalence, In Vitro Release and Associated Studies; Withdrawal. Federal Register. 17 May 2002;67(96):35122-35123.

14. N'Dri-Stempfer B, Navidi WC, Guy RH, Bunge AL. Improved bioequivalence assessment of topical dermatological drug products using dermatopharmacokinetics. Pharm Res, 26(2):316-328, 2009.

15. N'Dri-Stempfer B, Navidi W, Guy RH, Bunge AL. Optimising metrics for the assessment of bioequivalence between topical drug Products. Pharm Res, 25(7):1621-1630, 2008.

16. Kalia YN, Alberti I, Nabila S, Curdy C, Naik A, Guy RH. Normalization of stratum corneum barrier function and transepidermal water loss. Pharm Res, 17(9):1148-1150, 2000.

17. World Medical Association. WMA Declaration of Helsinki - Ethical Principles for Medical Research Involving Human Subjects. Available at: http://www.wma.net/en/30publications/10policies/b3/ index.html. Accessed 03/19, 2009.

18. Au WL, Skinner MF, Kanfer I. Comparison of tape stripping with the human skin blanching assay for the bioequivalence assessment of topical clobetasol propionate formulations. J Pharm PharmSci, 13(1):11-20, 2010.

19. Chow S-C, Wang H. On sample size calculation in bioequivalence trials. J Pharmacokinetic Pharmacodynam, 28(2):155-169, 2001.

20. Schuirmann DJ. A comparison of the Two One-Sided Tests Procedure and the Power Approach for assessing the equivalence of average bioavailability. J Pharmacokinet Biopharm, 15(6):657-680, 1987.

21. Bolton, S.; Bon, C., Pharmaceutical Statistics: Practical and Clinical Applications. 5th ed, Taylor and Francis, USA, 2009.

22. Reddy MB, Stinchcomb AL, Guy RH, Bunge AL. Determining dermal absorption parameters in vivo from tape strip data. Pharm Res, 19(3):292-298, 2002.

23. U.S. Department of Health and Human Services, Food and Drug Administration, Center for Drug Evaluation and Research. Guidance for Industry: Bioavailability and Bioequivalence Studies for Orally Administered Drug Products - General Considerations. Rockville: Food and Drug Administration; 2003.

24. Niazi SK. Handbook of Bioequivalence Testing. USA: Informa Healthcare Inc; 2007. Drugs and the Pharmaceutical Sciences, Vol 171. 